\title{
LOW-FIELD MAGNETORESISTANCE IN MANGANITE THIN FILMS
}

\begin{abstract}
E.S. VlakHov ${ }^{a, b}$, K. DöRR ${ }^{a}$, K.-H. MÜLLER ${ }^{a}$, A. HANDSTEIN ${ }^{a}$, K. Nenkov ${ }^{a, b *}$, T. Walter ${ }^{a}$, R.A. Chakalov ${ }^{c}$, R.I. Chakalova ${ }^{c}$ AND A.Y. SPASOV ${ }^{c}$

${ }^{a}$ Institut für Festkörper- und Werkstofforschung Dresden

P.O. Box 270016, 01171 Dresden, Germany

${ }^{b}$ International Laboratory of High Magnetic Fields and Low Temperatures Gajowicka 95, 53-529 Wrocław, Poland

'Institute of Electronics, Bulgarian Academy of Sciences, 1784 Sofia, Bulgaria

Grain boundaries play an important role in low-field magnetoresistance of $\mathrm{La}_{0.7} \mathrm{Ca}_{0.3} \mathrm{MnO}_{3}$ and $\mathrm{La}_{0.7} \mathrm{Sr}_{0.3} \mathrm{MnO}_{3}$ thin films deposited by magnetron sputtering and pulsed laser deposition on YSZ(100) and silicon substrates buffered by YSZ. Well-pronounced low-field magnetoresistance hysteresis was observed in magnetic fields applied in in-plane and out-of-plane directions. High values of local magnetoresistance sensitivity $\mathrm{d}(M R) / \mathrm{d} H$ in the vicinity of the coercive field were obtained reaching up to $0.2 \% / \mathrm{Oe}$ for $\mathrm{La}_{0.7} \mathrm{Ca}_{0.3} \mathrm{MnO}_{3}$ samples at $5 \mathrm{~K}$.
\end{abstract}

PACS numbers: 75.70.Ak, 73.50.Dn

Recently the colossal magnetoresistance (CMR) phenomenon has attracted much attention as both a fundamental research and an applied science challenge [1]. It has been demonstrated that low-field magnetoresistance (LFMR) effects have an important influence in bulk polycrystalline materials [2,3], polycrystalline thin films [4-6], and thin films with reduced epitaxy [7, 8]. It was established that the control of crystallinity perfection of thin manganite films, such as epitaxial strain or granularity, could be utilized for tuning of their MR properties, especially of LFMR [6-8]. In this paper, the influence of preparation conditions during magnetron sputtering (MS) or pulsed laser deposition (PLD) using various substrates on the resulting $\mathrm{MR}$ behaviour is investigated.

$\mathrm{La}_{0.7} \mathrm{Ca}_{0.3} \mathrm{MnO}_{3}$ (LCMO) thin films were prepared by magnetron sputtering and $\mathrm{La}_{0.7} \mathrm{Sr}_{0.3} \mathrm{MnO}_{3}$ (LSMO) thin films by PLD using ceramic targets (cf. Refs. $[5,6]$ ). The substrates were monocrystalline plates (i) of Y-stabilised $\mathrm{ZrO}_{2}$ (100) (YSZ) and (ii) of silicon buffered by YSZ layers. The structure of the deposited films was characterized by SEM and X-ray diffraction using Co $K_{\alpha}$ radiation. Magnetic measurements were carried out in an ac susceptometer and a SQUID magnetometer at fields up to $50 \mathrm{kOe}$. The resistivity was measured by the four-probe technique in a superconducting split-coil magnet at fields

*On leave from the Institute of Solid State Physics, BAS, 1784 Sofia, Bulgaria. 
up to $70 \mathrm{kOe}$ applied in parallel (in-plane) and perpendicular (out-of-plane) directions to the sample surface. The magnetoresistance ratio was determined by $M R=[(R(H)-R(0)] / R(0)$.

It was shown in our previous work $[5,6]$ that the deposition temperature and mismatch of the lattice constants between the manganite film and the substrate have a crucial influence on the film growth. For a very low lattice mismatch the obtained films are single crystalline independent of employed deposition technique. Here, the obtained LCMO films sputtered on YSZ substrates at a substrate temperature $T_{\text {sub }}=700^{\circ} \mathrm{C}$ are polycrystalline consisting of a mixture of (100) and (110) oriented grains with a size of about 50-100 nm. The influence of substrate temperature on the growth of LSMO thin films on YSZ substrates resulted in a gradual change of the grain orientation. Epitaxial growth has been found around $T_{\text {sub }}=800^{\circ} \mathrm{C}[6]$.

\section{TABLE}

Characteristic parameters of thin films: $\delta$ - thickness of the manganite layer, $T_{\text {peak }}$ - temperature of resistance maximum, $T_{\mathrm{C}}$ - Curie temperature.

\begin{tabular}{c|c|c|c|c}
\hline \hline Samples & Structure & $\delta[\mathrm{nm}]$ & $T_{\text {peak }}[\mathrm{K}]$ & $T_{\mathrm{C}}[\mathrm{K}]$ \\
\hline VZ3 & YSZ/LCMO & 208 & 199 & 225 \\
VZ10 & YSZ/LCMO & 100 & 271 & 265 \\
VZ12 & YSZ/LCMO & 50 & 266 & - \\
VZ13 & YSZ/LCMO & 25 & 266 & - \\
VSZ28 & Si/YSZ/LCMO & 200 & 246 & - \\
B & YSZ/LSMO & 76 & 150 & 360 \\
G & YSZ/LSMO & 76 & $>320$ & 356 \\
R & Si/YSZ/LSMO & 100 & 150 & 365
\end{tabular}

Table provides an overview of some characteristic parameters of representative films. In the low magnetic field region a well-pronounced hysteretic behaviour of resistance of $\mathrm{La}_{0.7} \mathrm{Ca}_{0.3} \mathrm{MnO}_{3}$ thin films was registered by in-plane and out-of-plane measurements as shown in Fig. 1.

Both runs of MR exhibit qualitatively the same behaviour - a peak in the low magnetic field region with a strong MR slope and nearly a constant small slope at higher fields. The peaks of MR for the in-plane case are located at lower fields and their amplitudes are higher. The analysis of the MR hysteresis for the case of in-plane measurement has shown that the field value of the MR peak (the so-called "switching" field $H_{\mathrm{sw}}$ ) practically coincides with that of the coercive field $H_{\mathrm{c}}$ (see Fig. 2a). Such behaviour was also found for the PLD La ${ }_{0.7} \mathrm{Sr}_{0.3} \mathrm{MnO}_{3}$ thin films obtained by deposition at lower substrate temperatures $\left(600-710^{\circ} \mathrm{C}\right)$. The reason for this behaviour is that carrier transport in a granular system becomes easier when the magnetic vectors of grains are ordered. Therefore, the resistivity should be a maximum near the coercive field $H_{\mathrm{c}}$, where the magnetization vanishes. 


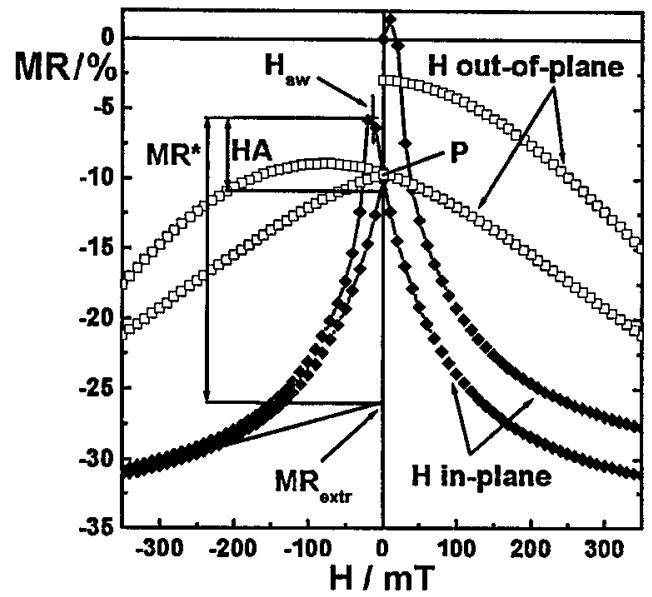

Fig. 1. Magnetoresistance ratio MR of the sample VZ3 measured at $T=20 \mathrm{~K}$. $\left(M R^{*}, M R_{\text {extr }}, H A\right.$, and $P$ are explained in the text).
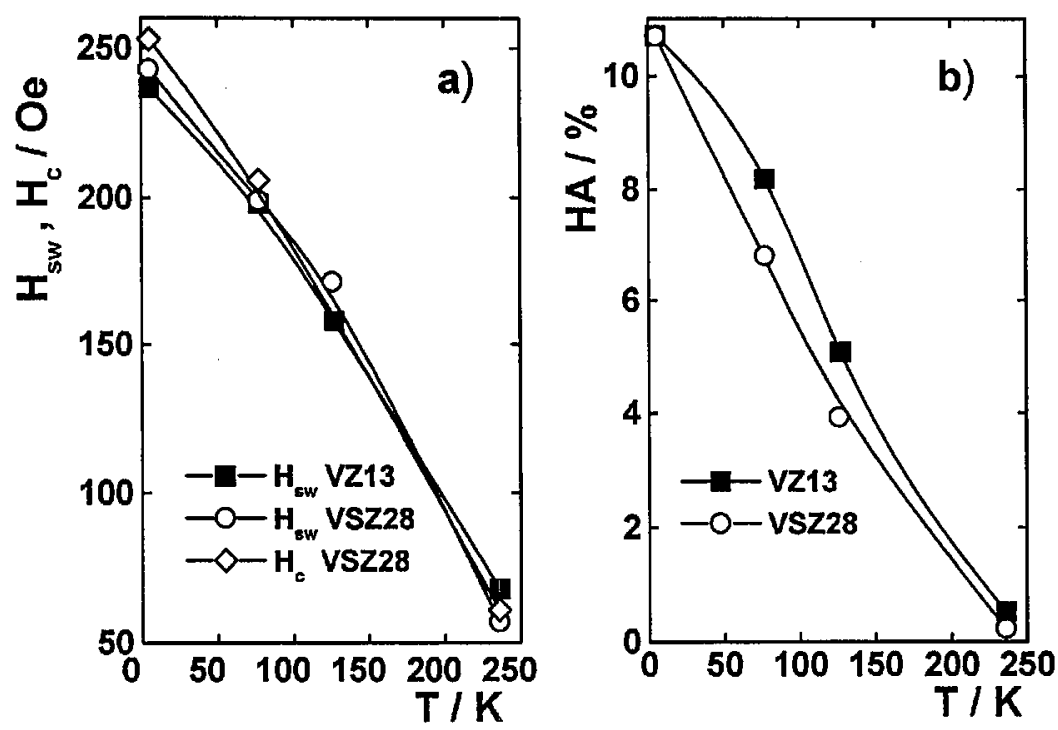

Fig. 2. Temperature dependences of (a) switching field $H_{\mathrm{sw}}$ and coercive field $H_{\mathrm{c}}$ and (b) hysteresis amplitude $H A=\Delta R\left(H_{\mathrm{sw}}\right) / R(0)$ of samples VZ13 and VSZ28.

The high value of the local $M R$ sensitivity $\mathrm{d}(M R) / \mathrm{d} H$ in the vicinity of the peak has to be noted, reaching values up to $0.2 \% / \mathrm{Oe}$ at $5 \mathrm{~K}$ for the $\mathrm{La}_{0.7} \mathrm{Ca}_{0.3} \mathrm{MnO}_{3}$ samples VZ13 and VSZ28. Compared with that, the high-field sensitivity of these samples is some orders of magnitude smaller, with values of approximately $0.5 \% / \mathrm{kOe}$. One can suppose that even higher values of LFMR sensitivity could be obtained in single layer films with a high degree of misoriented grains (for influence of misorientation angle at grain boundaries see [9]). 
It is noteworthy that the values of the low-field magnetoresistance amplitude $M R^{*}=\left[M R\left(H_{\mathrm{sw}}\right)-M R_{\mathrm{extr}}\right]$ are significantly influenced by the film thickness (where $M R\left(H_{\mathrm{sw}}\right)$ was determined at the switching field $H_{\mathrm{sw}}$ and $M R_{\text {extr }}$ is the zero field intercept of the linear high-field extrapolation of $M R$ ). Only a small influence of the film thickness on the values of $T_{\text {peak }}$ was observed as shown in Table (samples VZ10 to VZ13). For example, the $M R^{*}$ value of a sample decreases nearly by a factor of two in the temperature range of $T=5 \div 77 \mathrm{~K}$ by decreasing the film thickness from $100 \mathrm{~nm}$ (sample VZ10) down to $25 \mathrm{~nm}$ (sample VZ13). The maximal value, $M R^{*}(5 \mathrm{~K})=35 \%$, was registered for the $\mathrm{La}_{0.7} \mathrm{Ca}_{0.3} \mathrm{MnO}_{3}$ sample VZ10 (thickness $100 \mathrm{~nm}$ ) which exhibits the highest value of $T_{\text {peak }}$.

Together with $M R^{*}$ also the values of the hysteresis amplitude $H A$ strongly decrease with increasing temperature up to $T_{\mathrm{C}}$ as shown in Fig. $2 \mathrm{~b}$ ( $H A$ is the difference of MR values determined at $H_{\mathrm{sw}}$ and the crossing point $P$ as marked in Fig. 1). The temperature dependence of $H A$ is approximately linear. A similar strong decrease with temperature has been found for the spin polarisation of a LSMO thin film [10], that is clearly correlated with the observed decrease in low-field magnetoresistance of bulk material [2]. The reason for such behaviour of manganites is expected to be intrinsic. Therefore, new materials with higher $T_{\mathrm{C}}$ and weaker temperature dependence have been proposed.

In conclusion, the influence of extrinsic properties of manganite thin films on their LFMR has been investigated. A well-pronounced LFMR hysteresis was obtained in magnetic fields applied in the in-plane and out-of-plane directions. The high value of the sensitivity $\mathrm{d}(M R) / \mathrm{d} H$ in the vicinity of the coercive field (up to $0.2 \% / \mathrm{Oe}$ at $5 \mathrm{~K}$ ) has to be noted.

E.S.V. is grateful to the Deutsche Forschungsgemeinschaft (DFG) for financing the visit at IFW Dresden. This work was supported by the DFG (Sonderforschungsbereich 422).

\section{References}

[1] J.M.D. Coey, M. Viret, S. von Molnár, Adv. Phys. 48, 167 (1998).

[2] H.Y. Hwang, S.-W. Cheong, N.P. Ong, B. Batlogg, Phys. Rev. Lett. 77, 2041 (1996).

[3] P. Schiffer, A.P. Ramirez, W. Bao, S.-W. Cheong, Phys. Rev. Lett. 75, 3336 (1995).

[4] A. Gupta, G.Q. Gong, G. Xiao, P.R. Duncombe, P. Lecoeur, P. Trouilloud, Y.Y. Wang, V.P. Dravid, J.Z. Sun, Phys. Rev. B 54, R15629 (1996).

[5] E.S. Vlakhov, R.A. Chakalov, R.I. Chakalova, K.A. Nenkov, K. Dörr, A. Handstein, K.-H. Müller, J. Appl. Phys. 83, 2152 (1998).

[6] T. Walter, K. Dörr, K.-H. Müller, B. Holzapfel, D. Eckert, M. Wolf, D. Schläfer, L. Schultz, R. Grötzschel, Appl. Phys. Lett. 74, 2218 (1999).

[7] B.S. Teo, N.D. Mathur, S.P. Isaac, J.E. Evetts, M.G. Blamire, J. Appl. Phys. 83, 7157 (1998).

[8] J. O'Donnell, M. Onellion, M.S. Rzchowski, J.N. Eckstein, I. Bozovic, Phys. Rev. B 55, 5873 (1997).

[9] S.P. Isaac, N.D. Mathur, J.E. Evetts, M.G. Blamire, Appl. Phys. Lett. 72, 2038 (1998).

[10] J.-H. Park, E. Veskovo, H.-J. Kim, C. Kwon, R. Ramesh, T. Venkatesan, Phys. Rev. Lett. 81, 1953 (1998). 